\title{
The Interaction between Load Circuits and Decision of Frequency for Efficient Wireless Power Transfer
}

\author{
Kazuya Yamaguchi \\ Department of Control Engineering, National Institute of Technology, Nara College, Japan
}

\section{Article Info}

Article history:

Received: Dec 14, 2017

Revised: Feb 6, 2018

Accepted: Feb 22, 2018

Keyword:

wireless power transfer

coupling coefficient

state space representation

\begin{abstract}
This paper derives an expression of efficiency of wireless power transfer on a situation that there are two devices towards one AC power supply. The interaction between a power supply and load is paid attention on a conventional wireless power transfer system, in contrast, the interaction between loads must be taken account of on the situation too. This is attributed to a possibility that a load disturbs the energy transmitted from a power supply to another load. Moreover each load needs different frequency of power supply for the ideal transfer since they have different natural frequencies on many situations. This paper models a circumstance that there are a power supply and two loads with a state space equation, and proposes how to decide a frequency of power supply to realize efficient transfer for each load.
\end{abstract}

Copyright (c) 2018 Institute of Advanced Engineering and Science. All rights reserved.

\section{Corresponding Author:}

Kazuya Yamaguchi

Department of Control Engineering, National Institute of Technology, Nara College

22 Yata-cho, Yamatokoriyama, Nara, JAPAN

Email: k-yamaguchi@ctrl.nara-k.ac.jp

\section{INTRODUCTION}

Wireless Power Transfer (WPT) system is focused on the areas of energy, information, communication, and control, etc. In WPT, electric power is transferred on electromagnetic field by coils based on some electromagnetic theory. The basic theory of transfer electromagnetically was established by Nikola Tesla[1], and WPT is researched actively from the study of WPT on strongly coupled magnetic resonance[2].

The various methods are attempted according to some papers to improve efficiency and power, and to reduce energy loss of WPT. [3] focuses on circuit topology, and it mentions that the combination of series or parallel of circuit affects the intensity of power. A phase difference between the two transmission coils is controlled to increase efficiency[4]. The several coils are utilized for transmission of power to enlarge power, efficiency, and the distance between a power supply and loads[5][6].

Moreover WPT is applied for the various devices, places, and situations. In the medical area, a capsule endoscopy is charged from the outside of human body[7], and an effect to human body is investigated because the electromagnetic wave is generated on transmission wirelessly[8]. In the area of semiconductor, an application of WPT for the semiconductors is examined on low power and high frequency[9]. In terms of the long distance transmission, charge from a power supply on the ground to a balloon at high altitude is reviewed with electric propulsion[10].

As a merit of WPT, charging several devices simultaneously by closing them near a power supply is listed since WPT system does not need any connection of wire. However, when the device A is charged by a power supply, the other device B which is put near A may disturb exchanging energy between the power supply and $\mathrm{A}$. Therefore the interaction between devices must be investigated in addition to the transmission of energy from power supply to loads[11].

In this paper, the exchange of energy between a power supply and two loads is analyzed with a mathematical model based on modern control theory. Especially the coupling coefficients between a transmitting coil and device coil, and between device coils are focused for finding transportation efficiency. Finally, the effect of 
a load circuit which is not aimed to transfer energy is revealed, and the optimal angular frequency to maximize efficiency is obtained.

\section{DESIGN OF WPT CIRCUIT AND DERIVATION OF MATHEMATICAL MODEL}

At first, a circuit treated in this paper is shown as below.

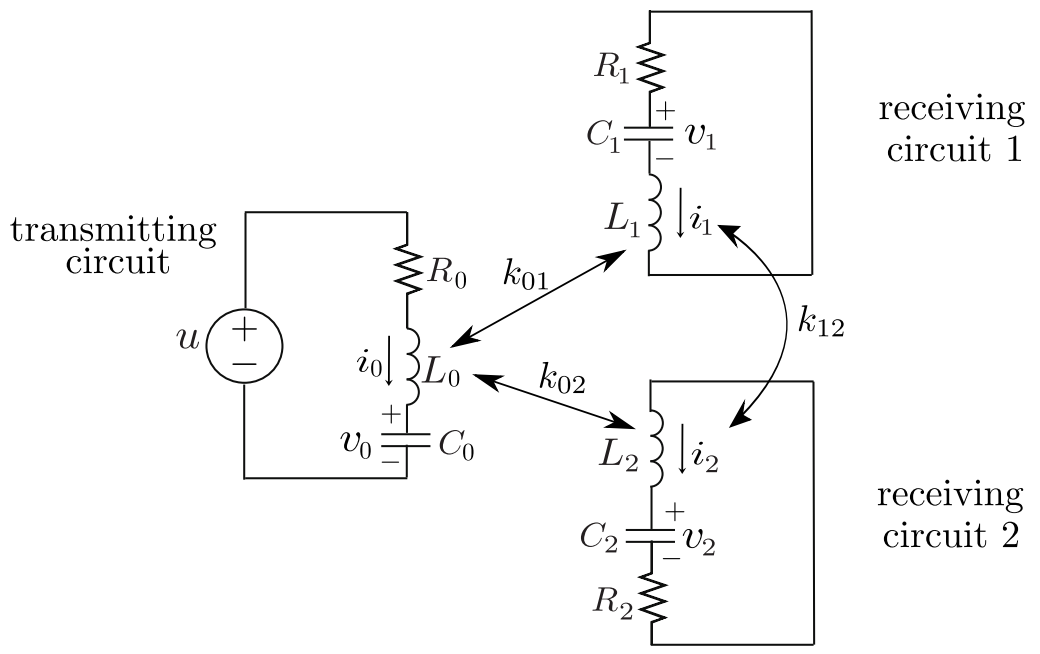

Figure 1. A WPT circuit which has two loads

In Figure 1, the left side is a transmitting circuit, and the circuits on the right side are receiving circuits, also two receiving circuits are distinguished as receiving circuit 1 and receiving circuit 2 each other. These three circuits are connected electromagnetically with three coils $L_{0}, L_{1}$, and $L_{2} . R_{0}$ is a parasitic resistance in the transmitting circuit, and $R_{1}$ and $R_{2}$ are the load resistances on each receiving circuit, and $C_{0}, C_{1}$, and $C_{2}$ are parasitic capacitances on each circuit. $k_{01}, k_{02}$, and $k_{12}$ are the coupling coefficients between each coil.

In this section, the optimal angular frequency of power supply which maximizes efficiency is derived. Then a mathematical model is found to derive the expressions of power and efficiency in the following [12].

$$
\begin{aligned}
\dot{x} & =A x+B u, x=\left[\begin{array}{lllllll}
v_{0} & v_{1} & v_{2} & i_{0} & i_{1} & i_{2}
\end{array}\right]^{\mathrm{T}} \\
A & =\frac{1}{\Delta}\left[\begin{array}{cccccc}
0 & 0 & 0 & \frac{\Delta}{C_{0}} & 0 & 0 \\
0 & 0 & 0 & 0 & \frac{\Delta}{C_{1}} & 0 \\
0 & 0 & 0 & 0 & 0 & \frac{\Delta}{C_{2}} \\
\Delta_{12} & \delta_{2} & \delta_{1} & \Delta_{12} R_{0} & \delta_{2} R_{1} & \delta_{1} R_{2} \\
\delta_{2} & \Delta_{02} & \delta_{0} & \delta_{2} R_{0} & \Delta_{02} R_{1} & \delta_{0} R_{2} \\
\delta_{1} & \delta_{0} & \Delta_{01} & \delta_{1} R_{0} & \delta_{0} R_{1} & \Delta_{01} R_{2}
\end{array}\right], B=\frac{1}{\Delta}\left[\begin{array}{c}
0 \\
0 \\
0 \\
-\Delta_{12} \\
-\delta_{2} \\
-\delta_{1}
\end{array}\right] \\
\Delta & =L_{0} L_{1} L_{2}\left(k_{01}^{2}+k_{02}^{2}+k_{12}^{2}-2 k_{01} k_{02} k_{12}-1\right) \\
\Delta_{01} & =L_{0} L_{1}\left(1-k_{01}^{2}\right) \\
\Delta_{02} & =L_{0} L_{2}\left(1-k_{02}^{2}\right) \\
\Delta_{12} & =L_{1} L_{2}\left(1-k_{12}^{2}\right) \\
\delta_{0} & =L_{0} \sqrt{L_{1} L_{2}}\left(k_{01} k_{02}-k_{12}\right) \\
\delta_{1} & =L_{1} \sqrt{L_{2} L_{0}}\left(k_{12} k_{01}-k_{02}\right) \\
\delta_{2} & =L_{2} \sqrt{L_{0} L_{1}}\left(k_{02} k_{12}-k_{01}\right) .
\end{aligned}
$$

This model is a state space equation to obtain the state solutions, and it has the state variables $v_{0}, v_{1}, v_{2}, i_{0}, i_{1}, i_{2}$, which are voltages and currents at each capacitor and coil. The above equation is solved with regard to these variables to find the state solutions, powers, and efficiencies. 


\section{FINDING OF EFFICIENCY FOR OBTAING THE OPTIMAL ANGULAR FREQUENCY}

\subsection{Derivation of the expressions of efficiencies}

By the mathematical model, a mathematical expression of efficiency is obtained. Efficiency is defined as the ratio of the load power and the input power, and two efficiencies must be distinguished because there are two loads in Figure 1. In this paper, the two efficiencies $\eta_{1}$ and $\eta_{2}$ are written as below.

$$
\begin{aligned}
\eta_{1} & =\frac{P_{1}}{P_{0}} \\
\eta_{2} & =\frac{P_{2}}{P_{0}} .
\end{aligned}
$$

Where $P_{0}$ is the power of power supply, and $P_{1}$ and $P_{2}$ are the load powers of $R_{1}$ and $R_{2}$ each other. From the equation (1), $\eta_{1}$ and $\eta_{2}$ are found theoretically, however it is difficult to describe all of the expression of $\eta_{1}$ and $\eta_{2}$, and therefore the second order approximation as regards $k_{01}, k_{02}$, and $k_{12}$ is applied because these values are less than 1 . The expressions of $\eta_{1}$ and $\eta_{2}$ with second order approximation are derived as follows.

$$
\begin{aligned}
& \eta_{1}=\frac{P_{1}}{P_{0}}=\frac{k_{01}^{2} R_{1} L_{0} L_{1} C_{1}^{2} \omega^{4}}{R_{0} L_{1}^{2} C_{1}^{2} \omega^{4}+R_{0}\left(R_{1}^{2} C_{1}-2 L_{1}\right) C_{1} \omega^{2}+R_{0}} \\
& \eta_{2}=\frac{P_{2}}{P_{0}}=\frac{k_{02}^{2} R_{2} L_{0} L_{2} C_{2}^{2} \omega^{4}}{R_{0} L_{2}^{2} C_{2}^{2} \omega^{4}+R_{0}\left(R_{2}^{2} C_{2}-2 L_{2}\right) C_{2} \omega^{2}+R_{0}} .
\end{aligned}
$$

$k_{12}$ is not contained in the expressions (3), hence it is obvious that its effect to efficiency is little compared to $k_{01}$ and $k_{02}$ in terms of efficiency. Moreover the optimal angular frequencies $\omega_{\text {opt1 }}$ and $\omega_{\text {opt } 2}$ which maximize $\eta_{1}$ or $\eta_{2}$ each other are obtained from expressions (3) as below.

$$
\begin{array}{r}
\omega_{\text {optn }}=\sqrt{\frac{2}{\left(2 L_{\mathrm{n}}-R_{\mathrm{n}}^{2} C_{\mathrm{n}}\right) C_{\mathrm{n}}}} \\
n=1 \text { or } 2
\end{array} .
$$

The desirable angular frequency for efficient WPT has been revealed in this section. Furthermore a numerical calculation is shown by the expression (4) in the following section.

\subsection{Numerical calculation of efficiencies}

In the former section, we find the optimal frequency by the mathematical model (1). In this section, the variation of efficiency with respect to angular frequency is investigated based on the expression (4). From the equation (3), a numerical calculation of $\eta_{1}$ and $\eta_{2}$ with practical values of circuit elements (Table 1) is shown in Figure. 2.

Table 1. values of elements

\begin{tabular}{|c|c|c|c|}
\hline$R_{0}$ & $1 \Omega$ & $C_{0}$ & $1 \mathrm{nF}$ \\
\hline$R_{1}$ & $10 \Omega$ & $C_{1}$ & $100 \mathrm{nF}$ \\
\hline$R_{2}$ & $100 \Omega$ & $C_{2}$ & $10 \mathrm{nF}$ \\
\hline$L_{0}$ & $1 \mu \mathrm{H}$ & $k_{01}$ & 0.2 \\
\hline$L_{1}$ & $10 \mu \mathrm{H}$ & $k_{02}$ & 0.2 \\
\hline$L_{2}$ & $70 \mu \mathrm{H}$ & $k_{12}$ & 0.2 \\
\hline
\end{tabular}




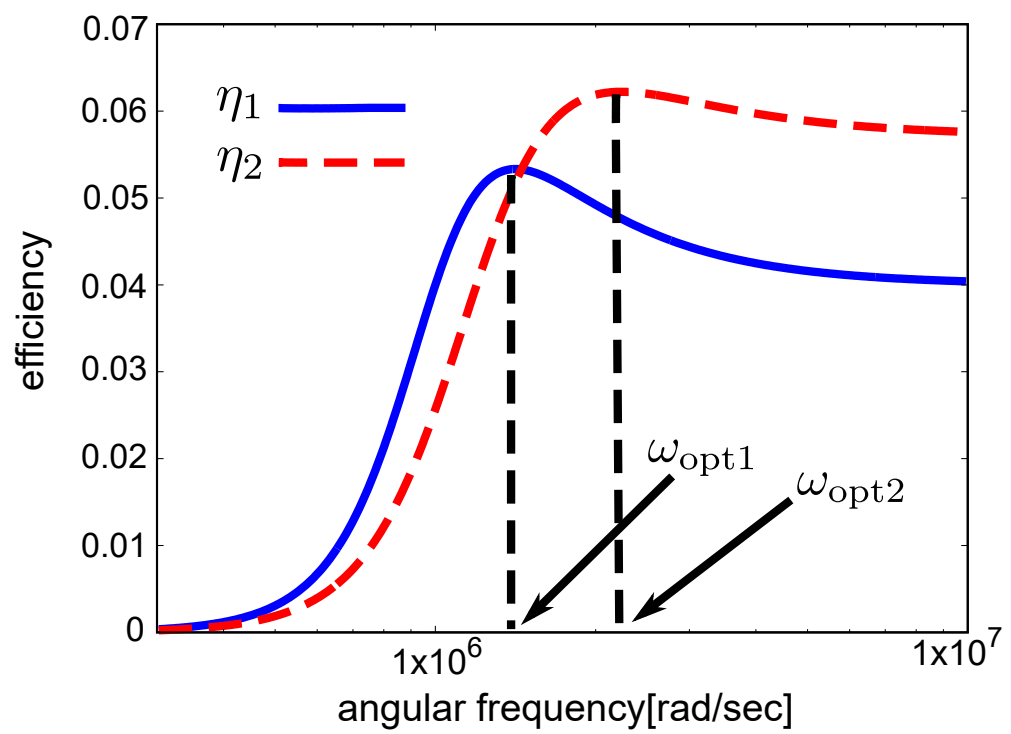

Figure 2. A numerical calculation of efficiency

By the expression (4), we find that $\omega_{\text {opt } 1}=1.41 \times 10^{6}[\mathrm{rad} / \mathrm{sec}], \omega_{\mathrm{opt} 2}=2.24 \times 10^{6}[\mathrm{rad} / \mathrm{sec}]$, and $\eta_{1}$ and $\eta_{2}$ are maximized with these angular frequencies each other. In many cases, the load circuits have its unique optimal frequency which maximizes efficiency, and therefore it is rare to maximize efficiencies from a power supply to the several loads simultaneously as shown in Figure 2. And it is seen that $\eta_{1}$ and $\eta_{2}$ converge to 0 at low frequency, and constant value at high frequency. The correctness is proved by taking the limit of the expression (3) with regard to $\omega$.

\section{CONCLUSION}

In this paper, we supposed the situation that there are two loads towards one power supply, and found the optimal angular frequency for efficient WPT. From the expression (4), it is obvious that the optimal angular frequency is not affected by the elements on transmitting circuit. Thus the elements in receiving circuits are significant when efficient WPT is desired, and the ideal frequency is different with each receiving circuit. Moreover the interaction between the coils on receiving circuits is little compared with the interaction between the coils on transmitting and receiving circuit. Hence the coupling between a transmitting coil and each receiving coil is more significant than the coupling between receiving coils.

\section{REFERENCES}

[1] N. Tesla, U. S. patent 1, 119, 732, 1914.

[2] A. Kurs, A. Karalis, R. Moffatt, J. D. Joannopoulos, P. Fisher, and M. Soljačić, "Wireless Power Transfer via Strongly Coupled Magnetic Resonances”, Science, vol. 317, pp. 83-86, 2007.

[3] Z. Bi, T. Kan, C. C. Mi, Y. Zhang, Z. Zhao, and G. A. Keoleian, "A review of wireless power transfer for electric vehicles: Prospects to enhance sustainable mobility", Applied Energy, vol. 179, pp. 413-425, 2016.

[4] A. Yoshida, E. Nishiyama, and I. Toyoda, "Basic Characteristics of a Selective Wireless Power Transfer System Using 4 Coils Aligned on a Line", The 67th Joint Conf. of Electrical, Electronics and Information Engineers in Kyushu, p. 13-1A-05, 2014.

[5] M. Kiani, U-M. Jow, and M. Ghovanloo, "Design and Optimization of a 3-Coil Inductive Link for Efficient Wireless Power Transmission", IEEE Transactions on Biomedical Circuits and Systems, vol. 5(6), pp. 579-591, 2011.

[6] X. Wang, X. Nie, Y. Liang, F. Lu, Z. Yan, and Y. Wang, "Analysis and experimental study of wireless power transfer with HTS coil and copper coil as the intermediate resonators system", Physica C: Superconductivity and its Applications, vol. 532, pp. 6-12, 2017.

[7] M. R. Basar, M. Y. Ahmad, J. Cho, and F. Ibrahim, "An improved resonant wireless power transfer system with optimumcoil configuration for capsule endoscopy”, Sensors and Actuators A: Physical, vol. 249, pp. 
207-216, 2016.

[8] J. Y. Mun, M. G. Seo, W. G. Kang, H. Y. Jun, Y. H. Park, J. K. Pack, "Study on the Human Effect of a Wireless Power Transfer Device at Low Frequency”, PIERS Proceedings, pp. 322-324, 2012.

[9] G. D. Capua, N. Femia, G. Petrone, G. Lisi, D. Du, and R. Subramonian, "Power and efficiency analysis of high-frequency Wireless Power Transfer", Electrical Power and Energy Systems, vol. 84, pp. 124-134, 2017.

[10] E. V. Wynsberghe, and A. Turak, "Station-keeping of a high-altitude balloon with electric propulsion and wireless power transmission: A concept study", Acta Astronautica, vol. 128, pp. 616-627, 2016.

[11] H. Kohara, and I. Toyoda, "Experimental Evaluation of Selective Wireless Power Transfer System Based on Coupled Magnetic Resonances", Record of 2012 Joint Conference of Electrical and Electronics Engineers in Kyushu, p. 02-1P-03, 2012.

[12] K. Yamaguchi, Y. Yamamoto, T. Hirata, E. Setiawan, and I. Hodaka, "Mathematical Expression of Optimal Frequencies for Wireless Power Transfer", Proceedings of The 3rd International Conference on Computer Engineering \& Mathematical Sciences, pp. 826-827, 2014. 